\title{
Necesidades de cuidadores familiares de personas con enfermedades huérfanas: cerrando brechas a través del cuidado enfermero
}

\author{
Family caregivers' needs of people with orphan diseases: closing arrows through \\ nursing care
}

\author{
Cielo Martínez Reyes ${ }^{1}$, Lizeth Oviedo López², Beatriz Polo Aragón ${ }^{3}$
}

\begin{abstract}
RESUMEN
Objetivo: establecer las necesidades de los cuidadores familiares de personas con enfermedades huérfanas teniendo en cuenta el Modelo de la Enfermería Adventista. Metodología: revisión de literatura que abarcó artículos cuantitativos y cualitativos hallados en bases de datos y revistas indexadas en idiomas español, inglés y portugués, entre los años 2014 y 2019, cuyo rigor metodológico cumplieran criterios STROBE, COREQ, PRISMA y JADAD. La información obtenida fue relacionada con el modelo de la Enfermería Adventista, NOC y NIC. Resultados: Se seleccionaron 28 artículos que analizaban necesidades físicas, psicológicas, sociales, educativas, espirituales, sanitarias y económicas en los cuidadores de personas con enfermedades huérfanas, que requerían soporte multidisciplinar e integral, incluyendo apoyo espiritual enfermero mediante intervenciones propuestas por la NIC y el modelo de Enfermería Adventista (cuidado, conexión con Dios y el otro, y empoderamiento del cuidador en su propia salud). Conclusión: Las necesidades de los cuidadores abarcan diferentes áreas del ser, que pueden ser abordadas con intervenciones independientes e interdependientes, identificadas mediante modelos teóricos.
\end{abstract}

Palabras clave: Cuidadores, Enfermedades Raras, Cuidado de Enfermería, Evaluación de Necesidades, Modelo Teórico.

\section{ABSTRACT}

Objective: establish the needs of family caregivers of people with orphan diseases considering the Adventist Nursing Model. Methodology: Literature review covering quantitative and qualitative articles found in databases and journals indexed in Spanish, English and Portuguese languages, between 2014 and 2019, whose methodological rigor met STROBE, COREQ, PRISMA, and JADAD criteria. The information obtained was related to the Adventist Nursing Model, NOC, and NIC. Results: 28 articles were selected that analyzed physical, psychological, social, educational, spiritual, health care and economic needs in caregivers of people with orphan diseases, which required multidisciplinary and integral support, including nurse spiritual support through interventions proposed by the NIC, and the Adventist Nursing model (care, connection with God and the other, and empowerment of the caregiver in their own health). Conclusion: the needs of caregivers cover different areas of being, which can be approached independently and interdependently, identified by theoretical models.

Keywords: Caregivers, Rare Diseases, Nursing Care, Needs Assessment, Theoretical Model.

\footnotetext{
${ }^{1}$ Universidad Cooperativa de Colombia, Medellín, Colombia. Orcid ID: 0000-0003-1545-5094

${ }^{2}$ Universidad Cooperativa de Colombia, Medellín, Colombia. Orcid ID: 0000-0002-7583-1162

${ }^{3}$ Universidad Cooperativa de Colombia, Medellín, Colombia.

Orcid ID: 0000-0003-1388-9518
} 


\section{INTRODUCCIÓN}

Las Enfermedades Huérfanas $(\mathrm{EH})$ son altamente debilitantes y traen consigo una alta tasa de morbimortalidad, alto grado de discapacidad y alteraciones en la calidad de vida, con un rasgo característico que es la baja o escasa prevalencia dentro la población (Avellaneda et al., 2007). La Unión Europea considera que una $\mathrm{EH}$ es aquella que no afecta a más de 5 personas de cada 10.000 habitantes y en concordancia con ello la Organización Mundial de la Salud (OMS) menciona que "la mayoría de los pacientes padece enfermedades que afectan a uno de cada 10.000 habitantes o menos"; así mismo, la Agencia Europea de Medicamentos, refiere que "hay entre 5.000 y 8.000 enfermedades que afectan entre 27 y 36 millones de personas" (OMS | Unidos para combatir las enfermedades raras, s. f.).

En Colombia, según el Ministerio de Salud y Protección Social "una EH es aquella crónicamente debilitante, grave, que amenaza la vida y con una prevalencia menor de 1 por cada 5.000 personas; comprenden, las enfermedades raras, las ultra huérfanas y olvidadas." Para el año 2013 se tenía un reporte de 13.238 casos (Enfermedades huérfanas, s. f.).

Las personas con $\mathrm{EH}$, se enfrentan a múltiples barreras debido a la complejidad de su proceso que genera alarmantes brechas para proporcionar el cuidado, como dificultad en obtener un diagnóstico exacto; opciones de tratamiento limitadas; poca o ninguna investigación disponible sobre su enfermedad; obstáculos para encontrar médicos o centros de tratamiento con experiencia en tratar una enfermedad específica; problemas para solicitar servicios médicos, sociales, asistenciales o financieros debido a la poca familiaridad sobre las enfermedades; falta de información; y sensación de aislamiento, pues por todo lo anterior, muchos son víctimas de rechazo y exclusión social. $(1,8)$

En el caso de Enfermería, también existe un analfabetismo generalizado en torno al tema y las necesidades de quienes las padecen, sus familias y cuidadores, hecho que limita la aplicación de un cuidado de calidad. Por ello se planteó como objetivo, establecer las necesidades de los cuidadores familiares de personas con enfermedades huérfanas teniendo en cuenta el Modelo de la Enfermería Adventista, a través de una revisión de literatura.

Para este modelo, la persona es un ser creado y diseñado por Dios -individuo, familia, comunidad, es un ser complejo -bio, psico, socio, cultural y espiritual- e interactivo con el propósito de conectarse con Dios, los seres humanos y toda la creación. La salud es integral, es decir, bienestar físico, mental, social, espiritual y cultural, incluye la comunión con Dios (JAE v79n5.pdf, s. f.). La Enfermería, es un llamado sagrado y servicio desinteresado que facilita el saneamiento y restauración del bienestar, los cuales son afectados por el entorno, visto como la reflexión de las leyes divinas de belleza y armonía (JAE v79n5.pdf, s. f.).

En concordancia con lo anterior, en la teoría se desarrollan tres constructos interconectados: cuidando, conectando y empoderando (ver figura 1). El cuidado es un valor fundamental, atributo poderoso y distintivo de la Enfermería, es atender más allá de lo ordinario, mediante la compasión (sensibilidad por el otro), competencia (conocimiento y habilidad), confianza (creer en los demás), conciencia (de lo moral), compromiso (intervenir a tiempo) y comportamiento (incluye el componente espiritual y actitud de interés por el otro). La conexión, es la interacción social, comunicación terapéutica, escucha activa y relación personal con Dios, para fortalecer al profesional en su vínculo con otros y ayudarles así a conectarse igualmente con el Ser Supremo a los recursos técnicos y humanos (JAE_v79n5. pdf, s. f.). El empoderar, es la capacidad de ejercer influencia sobre alguien o algo, es decir, toda acción encaminada por el profesional para que los pacientes cuiden de sí mismos, a través de la defensa, inspiración y motivación. La educación brindada por el enfermero cumple un rol importante en este concepto.

\section{METODOLOGÍA}

El presente estudio es una revisión integrativa de literatura.

\section{Criterios de Elegibilidad}

Artículos de investigaciones de acceso libre que abordaban el tema de interés: necesidades de los cuidadoresfamiliares de personas con $\mathrm{EH}$, escritos en inglés, español o portugués: cuantitativas (meta-análisis, estudios experimentales, cuasi experimentales, de cohortes, casos y controles, descriptivos), cualitativas (fenomenológicos, teorías fundamentadas, etnografías), excluyendo, artículos de reflexión y de opinión de expertos. Todos ellos hallados en bases de datos reconocidas y revistas indexadas y 
referenciadas en Publindex, Latindex y Scimago, con clasificación Q1, Q2, Q3, Q4, publicados entre los años 2014 y 2019.

Los preseleccionados, fueron evaluados con diferentes listas de chequeo y escalas de evaluación, según su tipo de estudio: STROBE (artículos de estudios observacionales), COREQ (artículos de estudios cualitativos), PRISMA (artículos de revisiones, revisiones sistemáticas, metaanálisis), y la escala de JADAD (artículos de ensayos clínicos controlados o experimentos); esto, para garantizar la elección de las investigaciones con diseños metodológicos robustos y de calidad, que permitan un nivel de evidencia y grados de recomendación elevados según Joanna Briggs Institute (JBI).

Finalmente, los hallazgos fueron relacionados y analizados a la luz del Modelo de Enfermería Adventista, los Nursing Outcomes Classification (NOC) y las Nursing Intervention Classification (NIC)

\section{Fuentes de Información y términos de búsqueda}

Bases de datos indexadas del área de la salud y las humanidades tales como EBSCO, SCIELO, DIALNET- PLUS, PUBMED, MEDLINE, SPRINGER LINK, DOAJ, BVS, CUIDEN, COCHRANE, ERIC, BASE-SEARCH, REDALYC.

Los descriptores utilizados para la búsqueda bibliográfica, incluyendo los booleanos "y" "o" "no" fueron: "cuidador enfermedad huérfana" y "necesidades cuidador enfermedad rara" y la combinación de ellas: cuidador enfermedad huérfana + necesidades cuidador enfermedad rara y sus equivalentes en inglés y portugués. Para el manejo de la bibliografía, se utilizó el gestor bibliográfico "Zotero".

\section{Manejo de Literatura Gris y Control de Sesgos}

Durante la búsqueda se encontraron publicaciones de estudios que aún no habían sido publicados en revistas, como tesis, excluidos en la selección. El seguimiento riguroso del proceso planteado en la revisión, escogiendo únicamente aquellos artículos que pasaron los diferentes filtros y cumplían con los parámetros establecidos en las diferentes listas y escalas de evaluación, garantizó el control de sesgos.

\section{RESULTADOS}

En la figura 1 se muestra que se hallaron 3.532 artículos que contenían las palabras clave. Al verificar el año de publicación (2014 - 2019), fueron excluidos 1.512, quedando así, 2.020. Posteriormente, al corroborar el tema de los artículos, fueron preseleccionados 159 que trataban sobre las necesidades de los cuidadores familiares de personas con EH. De ellos, se descartaron 13 artículos que no tenían acceso libre y 22 que correspondían a reflexiones y opiniones de expertos, por considerar que tienen bajo nivel de evidencia, según el JBI. De los 124 elegibles, 119 fueron publicados en revistas indexadas en Scimago, en categorías Q1, Q2, Q3 o Q4; de los mismos, 72 estaban repetidos, con lo cual, 47 documentos pasaron a la siguiente fase en la que se evaluaba su calidad metodológica a través de listas de chequeo y escalas de evaluación, tras lo cual quedaron finalmente 28.

Figura 1

Proceso de selección de los artículos a utilizar en la revisión de literatura.

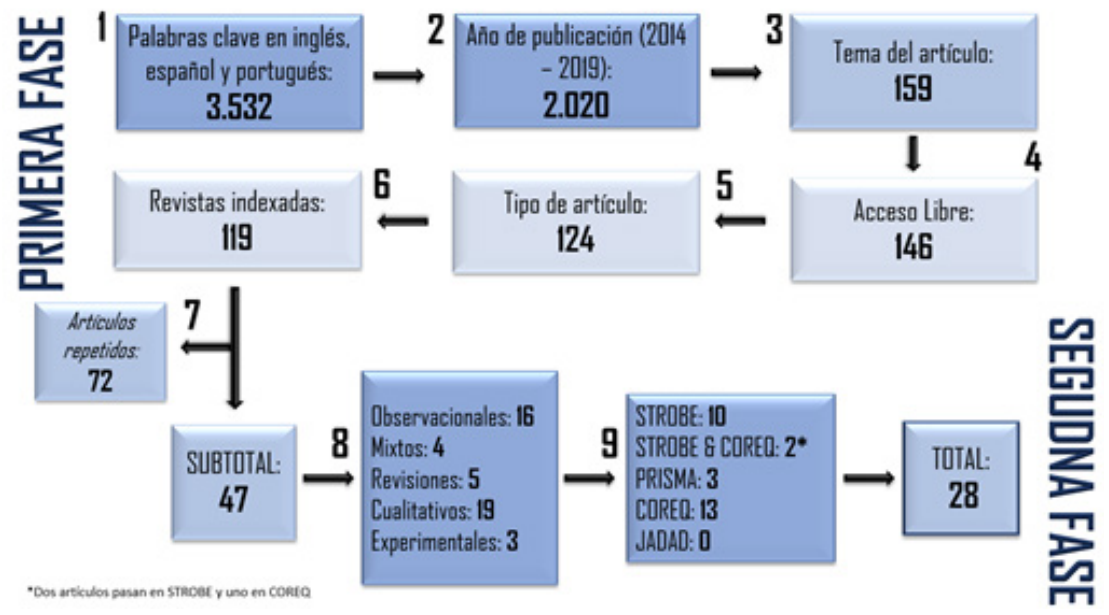

Fuente: Proceso de búsqueda y selección de artículos 
La mayor parte de los artículos seleccionados $(75 \%)$, se hallaron en el motor de búsqueda BVS. $89 \%$ publicados en inglés, que continúa siendo el más utilizado en la publicación de literatura científica en el área de la salud. Así mismo, el $43 \%$ y el $36 \%$ de los estudios fueron desarrollados en Europa y en Norteamérica respectivamente, mostrando entonces, que la cultura investigativa de estos continentes sobrepasa por mucho la cultura latina, teniendo Sudamérica un $7 \%$ de los estudios encontrados. La mayor parte (36\%) fueron publicados en 2018 y el $68 \%$ hacían parte de revistas indexadas en categoría Q1, lo cual denota su excelente calidad y rigor metodológico.

$46 \%$ fueron artículos de cualitativos, siendo más frecuentes las fenomenologías (11\%), con lo que se evidencia lo prolífica que se ha tornado la investigación cualitativa en el campo de la salud. Sin embargo, un 36\% fueron descriptivostransversales, y un $11 \%$ revisiones de literatura. $7 \%$ de los textos encontrados utilizaron una metodología mixta. Todas estas investigaciones fueron llevadas a cabo principalmente por enfermeros $(32 \%)$ y psicólogos $(21 \%)$, con formación doctoral en el $82 \%$ de los casos.
Entre las $\mathrm{EH}$ tratadas en estas publicaciones están: Distrofia Muscular de Duchenne, trastornos genéticos, neurológicos, del tejido conectivo, metabólicas e inmunodeficiencias; y se enfocaba principalmente en las necesidades de los cuidadores de pacientes no hospitalizados (78\%). Estos cuidadores en eran un $71 \%$ de los casos, mujeres, madres (43\%) o esposas $(11 \%)$ de los pacientes, lo que corrobora el rol de cuidado de enfermos, conferido a las mujeres, desde tiempos inmemoriales. En algunos artículos se reportó que el cuidador se encontrada a cardo de más de un familiar enfermo.

En la tabla 1, respecto a las necesidades de los cuidadores familiares de personas con enfermedades huérfanas, las señaladas en los artículos y que se relacionan con el Modelo de Enfermería Adventista fueron: aspectos físicos, psicológicos, sociales, educativos y espirituales. Además, también se encontraron otras variables como calidad de vida, carga del cuidador, aspectos de salud y económicos.

Tabla 1

Relación entre los hallazgos y el Modelo de Enfermería Adventista.

\begin{tabular}{|c|c|c|c|}
\hline $\begin{array}{l}\text { Necesi- } \\
\text { dades del } \\
\text { cuidador }\end{array}$ & Variable & Autores & Hallazgos \\
\hline \multirow[t]{3}{*}{ Físicas } & $\begin{array}{l}\text { Estado de salud general } \\
\text { del cuidador }\end{array}$ & $\begin{array}{l}\text { Mori Yuka (Mori et al., 2017), Landfeldt } \\
\text { Erik (Landfeldt et al., 2018) (Landfeldt et } \\
\text { al., 2016b), Stewart Michelle (Stewart et } \\
\text { al., 2018), Pelentsov Lemuel J. (Pelent- } \\
\text { sov et al., 2015), } \\
\text { Schwartz Carolyn E. (Schwartz et al., } \\
\text { 2017). }\end{array}$ & $\begin{array}{l}\text { Los problemas de salud física son } \\
\text { directamente proporcionales a la } \\
\text { dependencia de los niños. } \\
\text { Los cuidadores presentaron signos } \\
\text { y síntomas como dolor de cabeza, } \\
\text { pérdida de apetito y peso, mareos } \\
\text { o resfriados frecuentes, además de } \\
\text { problemas para realizar actividades } \\
\text { habituales. } \\
\text { Los cuidadores tenían antecedentes } \\
\text { personales de la misma enfermedad } \\
\text { que los pacientes.(Stewart etal., 2018) } \\
\text { (Pelentsov et al., 2015) }\end{array}$ \\
\hline & Cansancio & $\begin{array}{l}\text { Tejada Ortigosa Eva María (Tejada-Or- } \\
\text { tigosa et al., 2019), Mori Yuka (Mori et } \\
\text { al., 2017), Landfeldt Erik (Landfeldt et } \\
\text { al., 2018) (Landfeldt et al., 2016a), Utt- } \\
\text { ley Lesley (Uttley et al., 2018), Lauder } \\
\text { Bonnie (Lauder et al., 2018), Pelentsov } \\
\text { Lemuel J. (Pelentsov et al., 2016), Yoo } \\
\text { Jennie (Yoo et al., 2019), Schwartz Ca- } \\
\text { rolyn E. (Schwartz et al., 2017). }\end{array}$ & $\begin{array}{l}\text { Cansancio relacionado con la carga } \\
\text { del cuidador (Tejada-Ortigosa et al., } \\
\text { 2019) (Pelentsov et al., 2016) (Yoo } \\
\text { et al., 2019)(Mori et al., 2017). Hay } \\
\text { necesidad de un segundo cuidador, } \\
\text { expresó que así mejoraba la condición } \\
\text { física del cuidador, pero no contribuíaa } \\
\text { su salud emocional (Mori et al., 2017). }\end{array}$ \\
\hline & Descanso & $\begin{array}{l}\text { Landfeldt Erik (Landfeldt et al., 2018) } \\
\text { (Landfeldt et al., 2016a), Uttley Lesley } \\
\text { (Uttley et al., 2018), Egerod Ingrid (Ege- } \\
\text { rod et al., 2017), Pelentsov Lemuel J. } \\
\text { (Pelentsov et al., 2015), Ceña Domingo } \\
\text { Palacios (Palacios et al., 2018), Yoo Jen- } \\
\text { nie (Yoo et al., 2019), Schwartz Carolyn } \\
\text { E. (Stewart et al., 2018). }\end{array}$ & $\begin{array}{l}\text { Es innegable la mala calidad del sue- } \\
\text { ño y los problemas para dormir que } \\
\text { estaban relacionados con el estrés } \\
\text { y las hospitalizaciones del paciente } \\
\text { (Landfeldt et al., 2018)(Uttley et al., } \\
\text { 2018)(Egerod et al., 2017) lo cual, ha } \\
\text { generado impacto en la vida familiar. } \\
\text { (Palacios-Ceña et al., 2018) }\end{array}$ \\
\hline
\end{tabular}




\begin{tabular}{|c|c|c|c|}
\hline & Autocuidado & $\begin{array}{l}\text { Casas Claudia Patricia (Fundación } \\
\text { Universitaria de Ciencias de la Salud. } \\
\text { Hospital de San José. Bogotá, Colombia. } \\
\text { et al., 2018), Rice Danielle B. (Rice et } \\
\text { al., 2019), Stewart Michelle (Stewart et } \\
\text { al., 2018), Yoo Jennie (Yoo et al., 2019). }\end{array}$ & $\begin{array}{l}\text { Los cuidadores presentaban descuido } \\
\text { con su propia salud, ocasionando un } \\
\text { deterioro en la misma. }\end{array}$ \\
\hline \multirow[t]{6}{*}{ Psicológicas } & Soporte emocional & $\begin{array}{l}\text { Martín Raquel Alba (Alba-Martín, 2016), } \\
\text { Pelentsov Lemuel J. (Pelentsov et al., } \\
\text { 2015), Yoo Jennie (Yoo et al., 2019). }\end{array}$ & $\begin{array}{l}\text { El soporte emocional había sido insufi- } \\
\text { ciente para los cuidadores (Alba-Mar- } \\
\text { tín, 2016)(Pelentsov et al., 2015). Por } \\
\text { tanto, existe la necesidad manifestada } \\
\text { de recibir asesoramiento y apoyo } \\
\text { durante el proceso salud-enferme- } \\
\text { dad, en el diagnóstico, tratamiento y } \\
\text { hospitalizaciones. (Yoo et al., 2019) }\end{array}$ \\
\hline & Sufrimiento & $\begin{array}{l}\text { Egerod Ingrid (Egerod et al., 2017), } \\
\text { Soares Juliana de Lima (Soares et al., } \\
\text { 2016). }\end{array}$ & $\begin{array}{l}\text { El cuidador sufre debido a la situación } \\
\text { general de salud del ser querido y la } \\
\text { separación durante las hospitaliza- } \\
\text { ciones. }\end{array}$ \\
\hline & Soledad & $\begin{array}{l}\text { Kesselheim Aaron S (Kesselheim et } \\
\text { al., 2015), Egerod Ingrid (Egerod et al., } \\
\text { 2017), Lauder Bonnie (Lauder et al., } \\
\text { 2018), Pelentsov Lemuel J. (Pelentsov et } \\
\text { al., 2015) (Pelentsov et al., 2016). }\end{array}$ & $\begin{array}{l}\text { Las madres expresan que no reciben } \\
\text { apoyo de la comunidad y que hay } \\
\text { un sentimiento profundo de soledad } \\
\text { debido a la rareza de la enfermedad. } \\
\text { Se sienten incomprendidas por el } \\
\text { personal de salud quien las tilda de } \\
\text { molestas o demandantes al hacer } \\
\text { preguntas respecto a la condición } \\
\text { de su hijo (Lauder et al., 2018). Y a } \\
\text { pesar de estar rodeadas de amigos, } \\
\text { el sentimiento de soledad permanece. } \\
\text { (Egerod et al., 2017) }\end{array}$ \\
\hline & Estrés & $\begin{array}{l}\text { Landfeldt Erik (Landfeldt et al., 2018) } \\
\text { (Landfeldt et al., 2016b), Rice Danielle } \\
\text { B. (Rice et al., 2019), Uttley Lesley (Utt- } \\
\text { ley et al., 2018), Pelentsov Lemuel J. } \\
\text { (Pelentsov et al., 2015), Bose Mousumi } \\
\text { (Bose et al., 2019), Ceña Domingo Pa- } \\
\text { lacios (Palacios et al., 2018), Yoo Jennie } \\
\text { (Yoo et al., 2019), Schwartz Carolyn E. } \\
\text { (Schwartz et al., 2017). }\end{array}$ & $\begin{array}{l}\text { El estrés se presenta debido a la } \\
\text { condición del paciente, el tratamiento } \\
\text { y el resultado a largo plazo. También } \\
\text { se asocia las demandas de cuidar al } \\
\text { ser querido y tratar de cumplir con } \\
\text { otras responsabilidades familiares } \\
\text { o laborales (Landfeldt et al., 2016b) } \\
\text { (Bose et al., 2019) }\end{array}$ \\
\hline & Ansiedad & $\begin{array}{l}\text { Landfeldt Erik (Landfeldt et al., 2018) } \\
\text { (Landfeldt et al., 2016b), Rice Danielle B. } \\
\text { (Rice et al., 2019), Uttley Lesley (Uttley } \\
\text { et al., 2018), } \\
\text { Stewart Michelle (Stewart et al., 2018), } \\
\text { Pelentsov Lemuel J. (Pelentsov et al., } \\
\text { 2016), Soares Juliana de Lima (Soares } \\
\text { et al., 2016). }\end{array}$ & $\begin{array}{l}\text { La ansiedad está relacionada con el } \\
\text { avance de la enfermedad en el ser } \\
\text { querido, con no poder suplir las nece- } \\
\text { sidades del paciente-dolor, confort y } \\
\text { con una preocupación constante por } \\
\text { sus limitaciones (Rice et al., 2019). } \\
\text { También tiene que ver con los costos } \\
\text { de la enfermedad (Landfeldt et al., } \\
2018 \text { ) (Landfeldt et al., 2016b); y con } \\
\text { la preocupación por otros miembros } \\
\text { de la familia (Uttley et al., 2018), tanto } \\
\text { así que el } 20 \% \text { de los padres manifes- } \\
\text { taron tomar medicamentos para ello. } \\
\text { (Pelentsov et al., 2016) }\end{array}$ \\
\hline & Culpa & $\begin{array}{l}\text { S. Somanadhan (Somanadhan y Larkin, } \\
\text { 2016), Casas Claudia Patricia (Fun- } \\
\text { dación Universitaria de Ciencias de la } \\
\text { Salud. Hospital de San José. Bogotá, } \\
\text { Colombia. et al., 2018), Landfeldt Erik } \\
\text { (Landfeldt et al., 2018), Uttley Lesley } \\
\text { (Uttley et al., 2018), Lauder Bonnie } \\
\text { (Lauder et al., 2018), Pelentsov Lemuel } \\
\text { J. (Pelentsovetal., 2015), Bose Mousumi } \\
\text { (Bose et al., 2019), Baumbusch Jennifer } \\
\text { (Baumbusch et al., 2019). }\end{array}$ & $\begin{array}{l}\text { Se encontraron expresiones de cul- } \\
\text { pa al pensar que le transmitieron la } \\
\text { enfermedad a su hijo. (Landfeldt et } \\
\text { al., 2018)(Uttley et al., 2018)(Lauder } \\
\text { et al., 2018). Algunas madres se } \\
\text { sienten señaladas por la familia y los } \\
\text { miembros de la comunidad, incluso } \\
\text { por el personal médico (Baumbusch } \\
\text { et al., 2019) y manifestaron tener culpa } \\
\text { por no pasar suficiente tiempo con los } \\
\text { hijos sanos (Bose et al., 2019). }\end{array}$ \\
\hline
\end{tabular}




\begin{tabular}{|c|c|c|c|c|}
\hline & \multicolumn{2}{|l|}{ Incertidumbre } & $\begin{array}{l}\text { Somanadhan S. (Somanadhan y Larkin, } \\
\text { 2016), Martín Raquel Alba (Alba-Martín, } \\
\text { 2016), Rice Danielle B. (Rice etal., 2019), } \\
\text { Egerod Ingrid (Egerod et al., 2017), } \\
\text { Lauder Bonnie (Lauder et al., 2018), } \\
\text { Pelentsov Lemuel J. (Pelentsov et al., } \\
\text { 2016), Bose Mousumi (Bose et al., 2019), } \\
\text { Yoo Jennie (Yoo et al., 2019). }\end{array}$ & $\begin{array}{l}\text { La incertidumbre es generada por el } \\
\text { diagnóstico, tratamiento, resultados } \\
\text { a largo plazo y a la progresión de la } \\
\text { enfermedad - deterioro del niño -, lo } \\
\text { describen como un futuro incierto. } \\
\text { Además, por la responsabilidad de } \\
\text { tomar decisiones médicas difíciles } \\
\text { (Rice et al., 2019). }\end{array}$ \\
\hline & \multicolumn{2}{|l|}{ Miedo } & $\begin{array}{l}\text { Somanadhan S. (Somanadhan y Larkin, } \\
\text { 2016), Mori Yuka (Mori et al., 2017), Mar- } \\
\text { tín Raquel Alba (Alba-Martín, 2016), Rice } \\
\text { Danielle B. (Rice et al., 2019), Pelentsov } \\
\text { Lemuel J. (Pelentsov et al., 2015). }\end{array}$ & $\begin{array}{l}\text { La ansiedad está presente al recibir } \\
\text { el diagnóstico (Mori et al., 2017), el } \\
\text { tratamiento escaso y por el hecho que } \\
\text { la enfermedad no tiene cura; también, } \\
\text { tienen miedo a las complicaciones o } \\
\text { discapacidad que el niño pueda llegar } \\
\text { a sufrir (Somanadhan y Larkin, 2016) } \\
\text { (Alba-Martín, 2016) (Pelentsov et al., } \\
\text { 2015) y por saber que se quedarán } \\
\text { solos (Rice et al., 2019). }\end{array}$ \\
\hline & \multicolumn{2}{|l|}{ Depresión } & $\begin{array}{l}\text { Landfeldt Erik (Landfeldt et al., 2018) } \\
\text { (Landfeldt et al., 2016b), Cañedo M. (Ca- } \\
\text { ñedo et al., 2018), Uttley Lesley (Uttley et } \\
\text { al., 2018), Lauder Bonnie (Lauder et al., } \\
\text { 2018), Stewart Michelle (Stewart et al., } \\
\text { 2018), Pelentsov Lemuel J. (Pelentsov } \\
\text { et al., 2016). }\end{array}$ & $\begin{array}{l}\text { Desesperanza, tristeza y desespe- } \\
\text { ración por el estado del paciente, el } \\
\text { avance de la enfermedad y los costos } \\
\text { elevados. } 37 \% \text { de los cuidadores } \\
\text { estaban siendo tratados para la de- } \\
\text { presión, la cual, estaba relacionada } \\
\text { con la carga que tenían (Pelentsov } \\
\text { et al., 2016). }\end{array}$ \\
\hline & \multicolumn{2}{|l|}{ Afrontamiento } & $\begin{array}{l}\text { Somanadhan S. (Somanadhan y Larkin, } \\
\text { 2016), Martín Raquel Alba (Alba-Mar- } \\
\text { tín, 2016), Landfeldt Erik (Landfeldt et } \\
\text { al., 2018), Uttley Lesley (Uttley et al., } \\
\text { 2018), Lauder Bonnie (Lauder et al., } \\
\text { 2018), Magliano Lorenza (Magliano et } \\
\text { al., 2014), Boele Florien W. (Boele et } \\
\text { al., 2017), Bose Mousumi (Bose et al., } \\
\text { 2019), Schwartz Carolyn E. (Schwartz } \\
\text { et al., 2017). }\end{array}$ & $\begin{array}{l}\text { Los cuidadores utilizan medidas emo- } \\
\text { cionales, se obligan a permanecer con } \\
\text { una actitud positiva para mantener } \\
\text { unida a la familia, aunque lo consi- } \\
\text { deraron desafiante. Existe una lucha } \\
\text { emocional para aceptar el diagnóstico } \\
\text { y buscar opciones de tratamiento, el } \\
\text { deseo era mejorar la calidad de vida } \\
\text { del niño. Se comentó sobre la resilien- } \\
\text { cia y el crecimiento personal. }\end{array}$ \\
\hline \multirow[t]{4}{*}{ Sociales } & \multicolumn{2}{|c|}{ Recreación/Tiempo libre } & $\begin{array}{l}\text { Landfeldt Erik (Landfeldt et al., 2018) } \\
\text { (Landfeldt et al., 2016b) (Landfeldt et } \\
\text { al., 2016a), Rice Danielle B. (Rice et al., } \\
\text { 2019), Uttley Lesley (Uttley et al., 2018), } \\
\text { Stewart Michelle (Stewart et al., 2018), } \\
\text { Pelentsov Lemuel J. (Pelentsov et al., } \\
\text { 2015) (Pelentsov et al., 2016), Schwartz } \\
\text { Carolyn E. (Schwartz et al., 2017) }\end{array}$ & $\begin{array}{l}\text { Por la gran demanda de tiempo que } \\
\text { exige el cuidado del familiar, los } \\
\text { cuidadores no podian estar solos en } \\
\text { pareja, ni solos consigo mismos. Los } \\
\text { estudios expresaron pérdida de liber- } \\
\text { tad o espontaneidad debido a la vida } \\
\text { social reducida y poco tiempo para } \\
\text { realizar otras actividades. }\end{array}$ \\
\hline & \multicolumn{2}{|l|}{ Aislamiento } & $\begin{array}{l}\text { Pelentsov Lemuel J. (Pelentsov et al., } \\
\text { 2015), Baumbusch Jennifer (Baumbusch } \\
\text { etal., 2019), Yoo Jennie (Yoo et al., 2019) }\end{array}$ & $\begin{array}{l}\text { La situación de aislamiento es ge- } \\
\text { nerada por la condición rara del ser } \\
\text { querido. También, hay aislamiento } \\
\text { respecto a la familia pues el cuidador } \\
\text { principal permanece más tiempo en } \\
\text { el hospital (Yoo et al., 2019) }\end{array}$ \\
\hline & \multicolumn{2}{|l|}{ Estigma social } & $\begin{array}{l}\text { Somanadhan S. (Somanadhan y Larkin, } \\
\text { 2016), Casas Claudia Patricia (Fun- } \\
\text { dación Universitaria de Ciencias de la } \\
\text { Salud. Hospital de San José. Bogotá, } \\
\text { Colombia. et al., 2018), Uttley Lesley } \\
\text { (Uttley et al., 2018). }\end{array}$ & $\begin{array}{l}\text { Percepción de estigma del entorno, } \\
\text { reacción negativa de la sociedad, } \\
\text { rechazo, imprudencia, y descono- } \\
\text { cimiento. De hecho, las personas } \\
\text { asociaron la enfermedad con SIDA. } \\
\text { (Fundación Universitaria de Ciencias } \\
\text { de la Salud. Hospital de San José. } \\
\text { Bogotá, Colombia. et al., 2018) }\end{array}$ \\
\hline & $\begin{array}{l}\text { Redes de } \\
\text { apoyo }\end{array}$ & $\begin{array}{l}\text { Apoyo gu- } \\
\text { bernamen- } \\
\text { tal }\end{array}$ & $\begin{array}{l}\text { Tejada Ortigosa Eva María (Tejada-Or- } \\
\text { tigosa et al., 2019), Somanadhan S. } \\
\text { (Somanadhan y Larkin, 2016), Pelentsov } \\
\text { Lemuel J. (Pelentsov et al., 2016). }\end{array}$ & $\begin{array}{l}\text { Pocas son las personas que reciben } \\
\text { apoyo económico del gobierno. (Te- } \\
\text { jada-Ortigosa et al., 2019) Se espera } \\
\text { obtener mecanismos de apoyo de las } \\
\text { agencias gubernamentales. (Soma- } \\
\text { nadhan \& Larkin, 2016)(Pelentsov et } \\
\text { al., 2016) }\end{array}$ \\
\hline
\end{tabular}


Relaciones sociales

Relaciones familiares

Apoyo de organizaciones 0 fundaciones

Educativas Información recibida sobre la enfermedad
Somanadhan S. (Somanadhan y Larkin, 2016), Kesselheim Aaron S. (Kesselheim et al., 2015), Egerod Ingrid (Egerod et al., 2017), Lauder Bonnie (Lauder et al., 2018), Magliano Lorenza (Magliano et al., 2014), Boele Florien W. (Boele et al., 2017),

Stewart Michelle (Stewart et al., 2018)

Pelentsov Lemuel J. (Pelentsov et al., 2015) (Pelentsov et al., 2016),

Ceña Domingo Palacios (Palacios-Ceña et al., 2018), Baumbusch Jennifer (Baumbusch et al., 2019), Yoo Jennie (Yoo et al., 2019), Soares Juliana de Lima (Soares et al., 2016),

Tejada Ortigosa Eva María (Tejada-Ortigosa et al., 2019), Somanadhan S. (Somanadhan y Larkin, 2016), Casas Claudia Patricia (Fundación Universitaria de Ciencias de la Salud. Hospital de San José. Bogotá, Colombia. et al., 2018), Landfeldt Erik (Landfeldt et al., 2018), Uttley Lesley (Uttley et al., 2018), Kesselheim Aaron $S$. (Kesselheim et al., 2015), Lauder Bonnie (Lauder et al., 2018), Magliano Lorenza (Magliano et al., 2014), Stewart Michelle (Stewart et al., 2018),

Pelentsov Lemuel J. (Pelentsov et al., 2015) (Pelentsov et al., 2016), Bose Mousumi (Bose et al., 2019), Yoo Jennie (Yoo et al., 2019), Schwartz Carolyn E. (Schwartz et al., 2017).

Tejada Ortigosa Eva María (Tejada-Ortigosa et al., 2019), Casas Claudia Patricia (Fundación Universitaria de Ciencias de la Salud. Hospital de San José. Bogotá, Colombia. et al., 2018), Rice Danielle B. (Rice et al., 2019), Kesselheim Aaron S. (Kesselheim et al., 2015), Pelentsov Lemuel J. (Pelentsov et al., 2015) (Pelentsov et al., 2016).

Tejada Ortigosa Eva María (Tejada-Ortigosa et al., 2019), Somanadhan S. (Somanadhan y Larkin, 2016), Casas Claudia Patricia (Fundación Universitaria de Ciencias de la Salud. Hospital de San José. Bogotá, Colombia. et al., 2018), Rice Danielle B. (Rice et al., 2019), Kesselheim Aaron S. (Kesselheim et al., 2015), Egerod Ingrid (Egerod et al., 2017), Lauder Bonnie (Lauder et al., 2018), Pelentsov Lemuel J. (Pelentsov et al., 2015) (Pelentsov et al., 2016), Ceña Domingo Palacios (Palacios-Ceña et al., 2018), Baumbusch Jennifer (Baumbusch et al., 2019),
Los padres manifiestan que la cantidad de amigos se redujo; sin embargo, de los pocos que tenían recibían algún tipo de apoyo. (Pelentsov et al. 2016) A pesar de ello, el sentimiento de soledad permanecía. (Egerod et al., 2017) Los cuidadores usaron las redes sociales para comunicarse con otras personas en su situación, o que consideraron muy importante y una gran fuente de apoyo; también utilizaban este medio para buscar información sobre la enfermedad. Consideraron muy importante la necesidad de comunicarse con otros en su situación. (Somanadhan \& Larkin, 2016)(Kesselheim et al., 2015)(Lauder et al., 2018)(Baumbusch et al., 2019) (Yoo et al., 2019)

El 88\% de los familiares clave contaron con el apoyo de una pareja o cónyuge. (Magliano et al., 2014) Pero, con frecuencia la funcionalidad familiar y las relaciones de pareja se vieron afectadas. En algunos casos las madres se sintieron abandonadas por sus esposos en el cuidado del niño, algunas parejas se divorciaron y consideraron que la enfermedad del niño contribuyó a ese hecho (Somanadhan y Larkin, 2016), además, hay sensación de dejar al niño sano por fuera.

El pertenecer a organizaciones y/o fundaciones relacionadas con la enfermedad es una fuente importante de apoyo, porque aportan recursos de tipo económico, educativo, afectivo y sanitario (Fundación Universitaria de Ciencias de la Salud. Hospital de San José. Bogotá, Colombia. et al., 2018), pero muchos padres refieren dificultades para acceder a grupos de apoyo específico para la enfermedad de su hijo. (Pelentsov et al., 2016)

Hay cuidadores que reciben información comprensible y suficiente (Tejada-Ortigosa et al., 2019), pero este no es el común denominador; muchos, se ven abocados a buscar la información en sitios web de internet y en fuentes que se consideran no muy seguras, pues sentían frustración al no recibirla por parte del personal de salud. Y cuando la recibían, les parecía confusa y difícil de entender. Expresaron el deseo de que la sociedad también se nforme sobre el tema para disminuir el estigma social. 


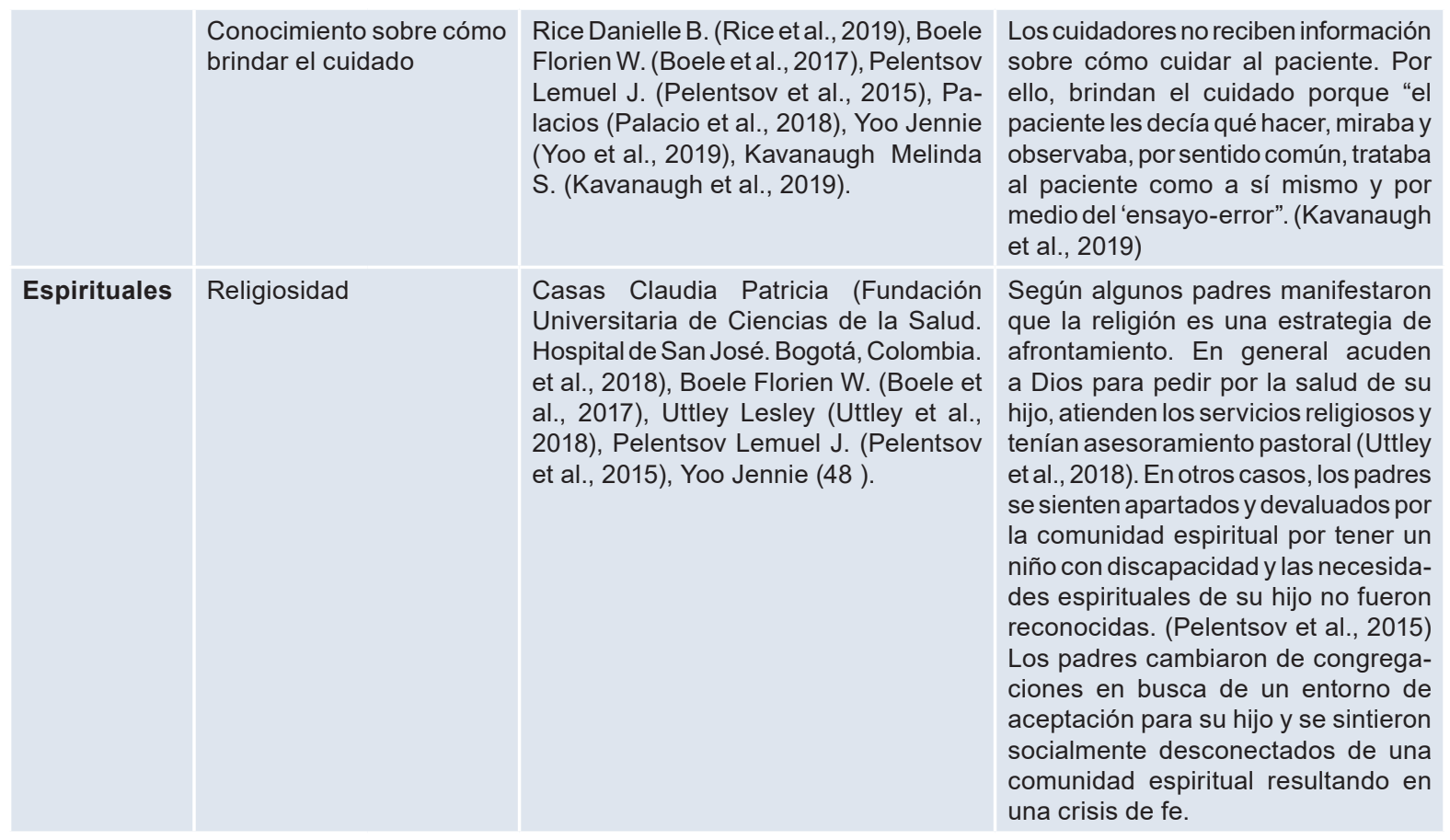

Fuente: Resultados de la búsqueda.

En la figura 2, Los hallazgos coinciden con lo planteado por el modelo de Enfermería Adventista en cuanto a la complejidad de la persona con componentes físicos, psicológicos, sociales, culturales y espirituales, que interactúa con su entorno que influye directamente en su estado de salud. Por tanto, es mandatorio que el profesional facilite una conexión con Dios, la cual primero, debe existir en él mismo; esto favorece un enlace que da lugar a un cuidado humano y consciente, que trasciende la satisfacción de necesidades meramente físicas, a las espirituales, sicológicas y sociales. En ese proceso, se debe lograr el empoderamiento del individuo, es decir, un cuidador que se cuide a sí mismo y no solo a su familiar enfermo.

Figura 2

Relación entre los componentes del Modelo de Enfermería Adventista y los resultados de la revisión de literatura.

Fuente: Autoría de los investigadores

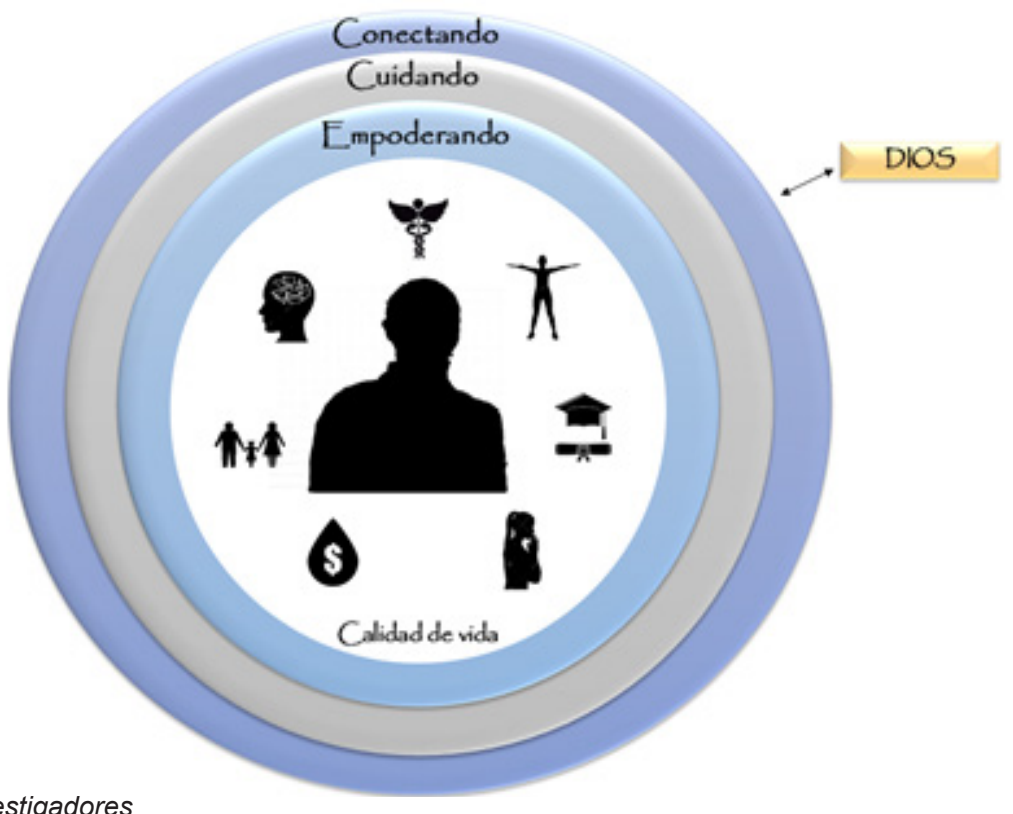


En la tabla 2 observamos que Para lograr lo anterior, el enfermero tiene como sustrato científico 51 NOC y 69 NIC, que guardan coherencia con las necesidades de los cuidadores de pacientes con enfermedades huérfanas.

Tabla 2

NOC y NIC relacionadas con las variables arrojadas en los resultados de la revisión de literatura.

\begin{tabular}{|c|c|c|c|}
\hline \multicolumn{4}{|c|}{ NOC Y NIC RELACIONADAS CON LAS VARIABLES } \\
\hline Necesidad del cuidador & Variable & NOC & $\mathrm{NIC}$ \\
\hline \multirow[t]{3}{*}{ Aspecto físico } & Descanso & $\begin{array}{l}\text { 1. Descanso }(0003) \\
\text { 2. Sueño }(0004)\end{array}$ & $\begin{array}{l}\text { 1. Mejorar el sueño (1850) } \\
\text { 2. Manejo de la energía (180) } \\
\text { 3. Aromaterapia (1330) } \\
\text { 4. Facilitar la meditación (5960) } \\
\text { 5. Terapia de relajación }(6040) \\
\text { 6. Manejo ambiental: confort (6482) } \\
\text { 7. Masaje (1480) } \\
\text { 8. Musicoterapia (4400) } \\
\text { 9. Prescribir medicación (2390) } \\
\text { 10. Baño (1610) } \\
\text { 11. Cuidados por relevo (7260) }\end{array}$ \\
\hline & $\begin{array}{l}\text { Estado de salud } \\
\text { general del cuidador }\end{array}$ & $\begin{array}{l}\text { 1. Nivel de fatiga (0007) } \\
\text { 2. Autocuidados: actividades de la } \\
\text { vida diaria ( } 0300) \\
\text { 3. Autocuidado: actividades instru- } \\
\text { mentales de la vida diaria (0306) } \\
\text { 4. Estado nutricional: ingestión } \\
\text { alimentaria y de líquidos (1008) } \\
\text { 5. Conducta de aumento de peso } \\
\text { (1626) } \\
\text { 6. Conducta de adhesión: dieta } \\
\text { saludable (1621) } \\
\text { 7. Estado de comodidad: física } \\
\text { (2010) } \\
\text { 8. Alteración del estilo de vida del } \\
\text { cuidador principal ( } 2203 \text { ) } \\
\text { 9. Salud física del cuidador princi- } \\
\text { pal (2507) }\end{array}$ & $\begin{array}{l}\text { 1. Fomento del ejercicio (0200) } \\
\text { 2. Alimentación (1050) } \\
\text { 3. Apoyo al cuidador principal } \\
\text { (7040) } \\
\text { 4. Asesoramiento nutricional } \\
\text { (5246) } \\
\text { 5. Enseñanza: dieta prescrita } \\
\text { (5614) } \\
\text { 6. Ayuda para ganar peso (1240) } \\
\text { 7. Monitorización nutricional (1160) } \\
\text { 8. Educación para la salud (5510) } \\
\text { 9. Cuidados por relevo ( } 7260)\end{array}$ \\
\hline & Autocuidado & $\begin{array}{l}\text { 1. Autocuidados: baño }(0301) \\
\text { 2. Autocuidados: vestir }(0302) \\
\text { 3. Autocuidados: comer }(0303) \\
\text { 4. Autocuidados: higiene }(0305) \\
\text { 5. Participación en el ejercicio } \\
\quad(1633)\end{array}$ & $\begin{array}{l}\text { 1. Ayuda con el autocuidado (1800) } \\
\text { 2. Ayuda con el autocuidado: baño/ } \\
\text { higiene (1801) } \\
\text { 3. Ayuda con el autocuidado: ali- } \\
\text { mentación (1803) } \\
\text { 4. Ayuda con el autocuidado: AIVD } \\
\text { (1805) }\end{array}$ \\
\hline \multirow[t]{4}{*}{ Aspecto psicológico } & Ansiedad & $\begin{array}{l}\text { 1. Nivel de ansiedad (1211) } \\
\text { 2. Autocontrol de la ansiedad } \\
\text { (1402) }\end{array}$ & $\begin{array}{l}\text { 1. Disminución de la ansiedad } \\
\text { (5820) } \\
\text { 2. Apoyo emocional ( } 5770) \\
\text { 3. Terapia de entretenimiento } \\
\text { (5360) } \\
\text { 4. Terapia de grupo ( } 5450) \\
\text { 5. Administración de medicación } \\
\text { (2300) } \\
\text { 6. Terapia asistida con animales } \\
\text { (4320) }\end{array}$ \\
\hline & Sufrimiento & 1. Severidad del sufrimiento (2003) & $\begin{array}{l}\text { 1. Dar esperanza }(5310) \\
\text { 2. Presencia }(5340)\end{array}$ \\
\hline & Depresión & $\begin{array}{l}\text { 1. Nivel de depresión (1208) } \\
\text { 2. Autocontrol de la depresión } \\
\text { (1409) }\end{array}$ & $\begin{array}{l}\text { 1. Control del estado de ánimo } \\
(5330) \\
\text { 2. Ayuda en la modificación de sí } \\
\text { mismo (4470) }\end{array}$ \\
\hline & Estrés & $\begin{array}{l}\text { 1. Nivel de estrés (1212) } \\
\text { 2. Conocimiento: manejo del es- } \\
\text { trés (1862) } \\
\text { 3. Factores estresantes del cuida- } \\
\text { dor familiar (2208) }\end{array}$ & $\begin{array}{l}\text { 1. Asesoramiento (5240) } \\
\text { 2. Técnica de relajación (5880) }\end{array}$ \\
\hline
\end{tabular}




\begin{tabular}{|c|c|c|c|}
\hline & Culpa & 1. Resolución de la culpa (1310) & $\begin{array}{l}\text { 1. Facilitar la expresión del senti- } \\
\text { miento de culpa ( } 5300) \\
\text { 2. Facilitar el perdón (5280) }\end{array}$ \\
\hline & Miedo & $\begin{array}{l}\text { 1. Nivel de miedo (1210) } \\
\text { 2. Autocontrol del miedo (1404) }\end{array}$ & $\begin{array}{l}\text { 1. Intervención en caso de crisis } \\
(6160)\end{array}$ \\
\hline & Soporte emocional & $\begin{array}{l}\text { 1. Aceptación: estado de salud } \\
\text { (1300) } \\
\text { 2. Salud emocional del cuidador } \\
\text { principal (2506) }\end{array}$ & $\begin{array}{l}\text { 1. Potenciación de la autoconcien- } \\
\text { cia }(5390)\end{array}$ \\
\hline & Afrontamiento & $\begin{array}{l}\text { 1. Afrontamiento de problemas } \\
(1302) \\
\text { 2. Resiliencia personal (1309) } \\
\text { 3. Afrontamiento de los problemas } \\
\text { de la familia (2600) }\end{array}$ & $\begin{array}{l}\text { 2. Mejorar el afrontamiento (5230) } \\
\text { 3. Contacto ( } 5460) \\
\text { 4. Fomentar la resiliencia ( } 8340) \\
\text { 5. Mejorar la autoconfianza (5395) }\end{array}$ \\
\hline & Soledad & 1. Severidad de la soledad (1203) & $\begin{array}{l}\text { 1. Potenciación de la socialización } \\
(5100)\end{array}$ \\
\hline Aspecto social & $\begin{array}{l}\text { Recreación/Tiempo } \\
\text { libre }\end{array}$ & $\begin{array}{l}\text { 1. Gestión del tiempo personal } \\
\text { (1635) }\end{array}$ & \\
\hline & Relaciones sociales & $\begin{array}{l}\text { 1. Estado de comodidad: sociocul- } \\
\text { tural }(2012) \\
\text { 2. Deterioro de la interacción so- } \\
\text { cial }(00052)\end{array}$ & $\begin{array}{l}\text { 1. Aumentar los sistemas de apoyo } \\
(5440) \\
\text { 2. Grupo de apoyo (5430) }\end{array}$ \\
\hline & $\begin{array}{l}\text { Relaciones fami- } \\
\text { liares }\end{array}$ & $\begin{array}{l}\text { 1. Lazos afectivos padres - hijo } \\
(1500) \\
\text { 2. Relación entre el cuidador prin- } \\
\text { cipal y el paciente ( } 2204)\end{array}$ & $\begin{array}{l}\text { 1. Estimulación de la integridad } \\
\text { familiar }(7100) \\
\text { 2. Fomentar la implicación familiar } \\
\text { ( } 7110) \\
\text { 3. Mantenimiento de procesos } \\
\text { familiares }(7130)\end{array}$ \\
\hline Aspecto educativo & $\begin{array}{l}\text { Información recibida } \\
\text { sobre la enfermedad }\end{array}$ & $\begin{array}{l}\text { 1. Conocimiento: proceso de la } \\
\text { enfermedad (1803) }\end{array}$ & $\begin{array}{l}\text { 1. Enseñanza: individual ( } 5606) \\
\text { 2. Enseñanza: proceso de enfer- } \\
\text { medad (5602) } \\
\text { 3. Enseñanza: procedimiento/ } \\
\text { tratamiento (5618) }\end{array}$ \\
\hline & $\begin{array}{l}\text { Conocimiento so- } \\
\text { bre cómo brindar } \\
\text { cuidado }\end{array}$ & $\begin{array}{l}\text { 1. Conocimiento: medicación } \\
\text { (1808) } \\
\text { 2. Conocimiento régimen terapéu- } \\
\text { tico (1813) } \\
\text { 3. Conocimiento procedimiento } \\
\text { terapéutico (1814) } \\
\text { 4. Conocimiento: cuidado de los } \\
\text { hijos (1826) } \\
\text { 5. Conocimiento: manejo de la } \\
\text { enfermedad crónica (1847) } \\
\text { 6. Preparación del cuidador fami- } \\
\text { liar domiciliario (2202) } \\
\text { 7. Rendimiento del cuidador prin- } \\
\text { cipal: cuidados directos (2205) }\end{array}$ & $\begin{array}{l}\text { 1. Enseñanza: medicamentos } \\
\text { prescritos (5616) } \\
\text { 2. Administración de medicación } \\
(2300) \\
\text { 3. Manejo de la medicación (2380) } \\
\text { 4. Facilitar el aprendizaje ( } 5520) \\
\text { 5. Educación parental: crianza } \\
\text { familiar de los niños (5566) }\end{array}$ \\
\hline Aspecto espiritual & Religiosidad & 1. Salud espiritual 2001 & $\begin{array}{l}\text { 1. Apoyo espiritual }(5420) \\
\text { 2. Facilitar el crecimiento espiritual } \\
(5426) \\
\text { 3. Facilitar la práctica religiosa } \\
\text { (5424) }\end{array}$ \\
\hline Calidad de vida & $\begin{array}{l}\text { Calidad de vida en } \\
\text { general }\end{array}$ & $\begin{array}{l}\text { 1. Calidad de vida (2000) } \\
\text { 2. Bienestar del cuidador principal } \\
(2508)\end{array}$ & \\
\hline Aspecto sanitario & $\begin{array}{l}\text { Oportunidad de } \\
\text { diagnóstico }\end{array}$ & $\begin{array}{l}\text { 1. Conocimiento: procedimientos } \\
\text { diagnósticos y terapéuticos } \\
\text { (1867) }\end{array}$ & \\
\hline & $\begin{array}{l}\text { Acceso a los servi- } \\
\text { cios de salud }\end{array}$ & $\begin{array}{l}\text { 1. Conocimiento: recursos sanita- } \\
\text { rios (1806) }\end{array}$ & $\begin{array}{l}\text { 1. Mejorar el acceso a la informa- } \\
\text { ción sanitaria ( } 5515 \text { ) } \\
\text { 2. Orientación en el sistema sani- } \\
\text { tario }(7400) \\
\text { 3. Educación para la salud ( } 5510) \\
\text { 4. Derivación ( } 8100)\end{array}$ \\
\hline
\end{tabular}




\begin{tabular}{|l|l|l|}
\hline $\begin{array}{l}\text { Apoyo del personal } \\
\text { sanitario }\end{array}$ & $\begin{array}{l}\text { 1. Participación de la familia en la } \\
\text { asistencia sanitaria profesional } \\
(2605)\end{array}$ & $\begin{array}{l}\text { 1. Apoyo en la toma de decisiones } \\
(5250)\end{array}$ \\
$\begin{array}{l}\text { 2. Planificación para el alta (7370) } \\
\text { 3. Escucha activa (4920) } \\
\text { 4. Intercambio de información de } \\
\text { cuidados de salud (7960) }\end{array}$ \\
\hline Gastos & $\begin{array}{l}\text { 1. Asistencia para los recursos } \\
\text { financieros (7380) }\end{array}$ \\
\hline
\end{tabular}

\section{DISCUSIÓN}

En cuanto a las necesidades físicas, se destaca el cansancio relacionado con la carga del cuidador. Al respecto, Tejada et al. (2019), Yoo et al. (2019), Mori et al., (2017) y por Pelentsov et al. (2016), afirman hay necesidad de un segundo cuidador, lo cual, mejora su condición física, aunque no contribuye a su salud emocional (Mori et al., 2017). De manera alarmante, otros autores reflexionan sobre la mala calidad del sueño y los problemas para dormir que estaban relacionados con el estrés (Landfeldt et al., 2018)(Uttley et al., 2018)(Egerod et al., 2017) y hospitalizaciones del paciente (Egerod et al., 2017), cuyo impacto, genera cambios en la dinámica y vida familiar. (Palacios-Ceña et al., 2018)

En este sentido, el déficit de autocuidado referido por Casas (Fundación Universitaria de Ciencias de la Salud. Hospital de San José. Bogotá, Colombia. et al., 2018), Rice et al. (2019), Stewart et al. (2018) y Yoo et al. (2019), tiene repercusión en el estado de salud general del cuidador, evidenciado en hallazgos como abandono de la propia salud, trayendo como consecuencia cefalea, pérdida de apetito y peso, mareos o resfriados frecuentes y problemas para realizar actividades habituales. Ante estas situaciones, el modelo de Enfermería Adventista, sostiene que la recuperación física va de la mano con la esperanza, la fe para ver claramente perspectivas de cómo resolver un problema, por ello el profesional está llamado a cuidar más allá de lo ordinario (JAE_v79n5.pdf, s. f.).

Con respecto a las necesidades psicológicas, Landfeldt et al. (2018), Rice et al. (2019), Uttley et al. (2018), Stewart et al. (2018) y Soares et al. (2016), sostienen que el estrés, incertidumbre, culpa, depresión, ansiedad y miedo, asociados al estado del ser querido, su diagnóstico, tratamiento y pronóstico, afecta la salud mental de los cuidadores. En los estudios de Kesselheim et al. (2015), Egerod et al. (2017) y Pelentsov et al. (2015) Pelentsov et al. (2016), las mujeres expresaron que no recibieron apoyo de la comunidad y que había un sentimiento profundo de soledad debido a la rareza de la enfermedad, lo anterior respaldado por Lauder et al. (2018), quien también indicó que las madres se sentían incomprendidas por el personal de salud que las tildaba de molestas o demandantes al hacer preguntas respecto a la condición de su hijo, actitudes alarmantes del enfermero, que aumentan sentimientos de soledad, incertidumbre y sufrimiento, lo cual desvela una gran necesidad de conexión con Dios, para alcanzar la sanidad, el crecimiento y la plenitud (JAE_v79n5.pdf, s. f.).

A propósito, dos temas prioritarios emergieron en la revisión: soporte emocional y afrontamiento. Respecto al primero, Yoo et al. (2019), encontró la necesidad manifestada de recibir asesoramiento y apoyo durante el proceso salud-enfermedad, en el diagnóstico, tratamiento y hospitalizaciones. En cuanto al segundo, Somanadhan y Larkin, (2016), Landfeldt et al. (2018), Uttley et al. (2018), Magliano et al. (2014), Boele et al. (2017) y otros, enfatizaron que los cuidadores afrontan la situación sin apoyo psicológico y de manera empírica, obligándose a permanecer con una actitud positiva para mantener unida a la familia, lo cual se constituye en todo un desafío y una lucha emocional en la aceptación del diagnóstico y búsqueda de opciones de tratamiento. Este vacío en el trabajo multidisciplinar y el de Enfermería, establece otra brecha que el cuidado puede estrechar por medio de una conexión efectiva entre el cuidador y demás profesionales de la salud (JAE_v79n5.pdf, s. f.).

Otras necesidades, las sociales, se enmarcan en la carencia de recreación-tiempo libre y redes de apoyo. Por la gran demanda de tiempo que exige el cuidado del familiar, los cuidadores no podían estar solos en pareja, ni solos consigo mismos. Además, se evidenció pérdida de libertad o espontaneidad debido a la vida social reducida y poco tiempo para realizar otras actividades. Según Pelentsov et al. (2016), la red de amigos es reducida, pero brinda apoyo. A pesar de ello, Egerod et al. (2017) observó que, en ocasiones, el sentimiento de soledad permanecía. Surgió también la sensación de aislamiento y estigma social por la condición 
rara del ser querido. Yoo et al. (2019), indicó que había aislamiento del cuidador con la misma familia, porque debía permanecer más tiempo en el hospital. La percepción de estigma del entorno, reacción negativa de la sociedad y rechazo, tenía como causa la imprudencia y desconocimiento de la sociedad. De hecho, Casas (Fundación Universitaria de Ciencias de la Salud. Hospital de San José. Bogotá, Colombia. et al., 2018) resalta que las personas asocian la enfermedad con el SIDA. Esto indica la urgencia de educación.

Desde otra perspectiva, Magliano et al. (2014) observó que el $88 \%$ de los familiares clave contaron con el apoyo de una pareja o cónyuge. Pero los otros autores, sostuvieron que la funcionalidad familiar y las relaciones de pareja se vieron afectadas. En algunos casos, las madres se sintieron abandonadas por sus esposos en el cuidado del niño y se presentaron divorcios, cuya causa fue atribuida a la $\mathrm{EH}$. Somanadhan y Larkin, (2016), agrega que los padres cuidadores sienten que excluyen al hijo sano.

Es en condiciones como las descritas, que el enfermero debe recordar que todos existen y funcionan en un ambiente que abarca los aspectos naturales, socioculturales, políticos y materiales y que más allá de los contextos individuales y ambientales se encuentra el poder abarcador de Dios, el Creador de los seres humanos, del ambiente $y$, en efecto, del universo, creencia que da soporte emocional y espiritual (JAE v79n5.pdf, s. f.). Los cuidadores estimaron que el pertenecer a organizaciones y/o fundaciones relacionadas con la enfermedad es una fuente importante de apoyo económico (Tejada et al., 2019), educativo, afectivo y sanitario (Fundación Universitaria de Ciencias de la Salud. Hospital de San José. Bogotá, Colombia. et al., 2018). Pero no todos cuentan con tal soporte (Pelentsov et al., 2016) (Somanadhan y Larkin, 2016).

Por su parte, las necesidades educativas, fueron el común denominador a lo largo de los diferentes estudios, toda vez que los cuidadores familiares estaban deseosos de conocimientos sobre la enfermedad y la atención que requieren sus seres queridos. Yoo et al. (2019) Rice et al. (2019). Mientras que Tejada et al. (2019) describió que, para los cuidadores, la información recibida sobre la enfermedad fue compresible y suficiente, otros autores como Casas et al. (2018), Kesselheim et al., (2015) y Egerod et al. (2017) indicaron que fue considerada devastadora, deficiente y confusa. Esto los obliga a acudir al internet como fuente de información (Palacios-Ceña et al., 2018).
Kavanaugh et al. (2019) confirmó que los cuidadores que no habían recibido educación ofrecían el cuidado porque "el paciente les decía qué hacer, miraban y observaban, por sentido común, trataba al paciente como a sí mismos y por medio del "ensayo-error".

Los cuidadores familiares se ven enfrentados a diversos escenarios emocionales, los cuales afectan negativamente las dinámicas de la propia vida debido a la falta de información referente a la enfermedad y a los conocimientos para brindar el cuidado en los hogares. Por tanto, desde la Enfermería cuidar a quien cuida, con acciones científicamente demostradas, refleja el corazón y el alma de la disciplina. (JAE_v79n5.pdf, s. f.)

Pese a que las necesidades espirituales están implícitas en el ser y por tango el no atenderlas puede hacer mella en la salud del cuidador, fue el tema menos tratado en la literatura seleccionada. Uttley et al., (2018) denotó que algunos padres se apoyan en la religión como una estrategia de afrontamiento, mientras que Pelentsov et al. (2015) encontró que los padres se sintieron apartados y devaluados por la comunidad espiritual por tener un niño con discapacidad, por lo que las necesidades en este sentido no fueron reconocidas. También indicó que los padres cambiaron de congregaciones en busca de un entorno de aceptación para su hijo y se sintieron socialmente desconectados, generando una crisis de fe. Ante esta realidad, el enfermero, debe tener en cuenta que el cuidado emana de un profundo respeto por el individuo como hijo de Dios, y un profundo deseo de mostrar Su amor a la persona necesitada, tal como se asevera en uno de los constructos clave del modelo de Enfermería Adventista: "conectando" (conectar con Dios y conectar con el otro).

Por otro lado, las necesidades sanitarias se evidenciaron en diferentes perspectivas, desde la que resalta el soporte que proporciona el equipo multidisciplinario, como lo afirmó Soares et al. (2016) y Boele et al. (2017), como la que alerta sobre la insensibilidad, desconsideración, falta de empatía y conocimientos, como lo ratifican Bose et al. (2019) y Rice et al. (2019). El abordaje diagnóstico de la enfermedad fue descrito como una experiencia complicada, por la manifestación de los síntomas y ejecución de múltiples pruebas que en diversos casos causaron agotamiento e incertidumbre (Palacios-Ceña et al., 2018) y (Baumbusch et al., 2019). El sistema de salud, el acceso a los servicios y al tratamiento tienen obstáculos: encontrar un lugar con la atención y el nivel de complejidad requeridos, las grandes 
distancias para recibir los servicios, cobertura limitada y alto costo (Somanadhan y Larkin, 2016), (Casas et al., 2018) (Baumbusch et al., 2019), situaciones que ponen de manifiesto necesidades económicas que se constituyen en una carga adicional, como lo aclaran Stewart et al., (2018), Kesselheim et al. (2015) y Pelentsov et al. (2016), por el estrés y angustia que generan (Pelentsov et al., 2016). Esta situación se agrava porque los padres se ven obligados a reducir las horas de trabajo e incluso dejarlo para dedicarse al cuidado del familiar (Landfeldt et al., 2016) Esto obliga a un solo familiar a asumir la responsabilidad financiera y aumentar sus horas de trabajo para hacer frente a ese aspecto. Además de ello, (Tejada et al. (2019) referenció que los cuidadores no tienen apoyo para compatibilizar su vida laboral. No obstante, Boele et al. (2017) manifestó que algunos cuidadores recibieron apoyo en el trabajo, lo cual puede convertirse en elemento positivo, porque pudieron desempeñarse en su entorno laboral y de igual forma contar con un espacio para el cuidado de su ser querido y solventar las necesidades financieras.

Finalmente, se evidenció que la calidad de vida de los cuidadores primarios se vio disminuida desde la aparición de la enfermedad del familiar, tanto por el estado de salud de los pacientes (Landfeldt et al., 2018) (Landfeldt et al., 2016), como por el del cuidador (Linertová et al., 2019). Así, quienes cuidan, se vuelven nuevos sujetos de cuidado. En este contexto, cabe destacar que la Enfermería busca contribuir a la inclusión y protección de los cuidadores familiares, ya que son vistos como un binomio, y el cuidado realizado debe ser integral, por lo que al intervenir cada componente del ser de manera individual se está contribuyendo directamente a la calidad de vida del ser, tal y como lo plantea el Modelo de Enfermería Adventista, con cuidar, conectar y empoderar (JAE_v79n5.pdf, s. f.).

Declaración de financiamiento y de conflictos de interés:

El estudio fue financiado por los autores, quienes declaran no tener conflictos de interés

\section{Correspondencia:}

Cielo Martínez Reyes

Correo electrónico:

cielorebe26@hotmail.com
Lizeth Oviedo López

Correo electrónico:

loviedo@unac.edu.co

Beatriz Eliana Polo Aragón

Correo electrónico:

elipolo@unac.edu.co

\section{REFERENCIAS BIBLIOGRÁFICAS}

Alba, R. (2016). Síndrome de Mowat-Wilson: Historia de vida de la fortaleza de una madre. Revista Científica de la Sociedad Española de Enfermería Neurológica, 44, 25-30. Recuperado de: https:// doi.org/10.1016/j.sedene.2016.06.002

Avellaneda, A., Izquierdo, M., Torrent, J., y Ramón, J. R. (2007). Enfermedades raras: Enfermedades crónicas que requieren un nuevo enfoque sociosanitario. Anales del Sistema Sanitario de Navarra, 30(2). Recuperado de: https://doi. org/10.4321/S1137-66272007000300002

Baumbusch, J., Mayer, S. y Sloan-Yip, I. (2019). Alone in a Crowd? Parents of Children with Rare Diseases' Experiences of Navigating the Healthcare System: XXXX. Journal of Genetic Counseling, 28(1), 80-90. Recuperado de : https://doi.org/10.1007/s10897-018-0294-9

Boele, F. W., van Uden-Kraan, C. F., Hilverda, K., Weimer, J., Donovan, H. S., Drappatz, J., Lieberman, F. S., Verdonck-de Leeuw, I., \& Sherwood, P. R. (2017). Neuro-oncology family caregivers' view on keeping track of care issues using eHealth systems: It's a question of time. Journal of Neuro-Oncology, 134(1), 157-167. Recuperado de: https://doi.org/10.1007/s11060017-2504-y

Bose, M., Mahadevan, M., Schules, D. R., Coleman, R. K., Gawron, K. M., Gamble, M. B., Roullet, J.-B., Gibson, K. M., \& Rizzo, W. B. (2019). Emotional experience in parents of children with Zellweger spectrum disorders: A qualitative study. Molecular Genetics and Metabolism Reports, 19, 100459. Recuperado de: https://doi.org/10.1016/j. ymgmr.2019.100459

Cañedo, M., Rice, D. B., Levis, B., Carrier, M.-E., Cumin, J., Malcarne, V. L., Hagedoorn, M., Thombs, B. D., \& the Scleroderma Caregiver Advisory Committee. (2018). Factors associated with symptoms of depression among informal caregivers of people with systemic sclerosis: A cross-sectional study. Disability and Rehabilitation, 1-6. Recuperado de: https://doi.or g/10.1080/09638288.2018.1500647 
Egerod, I., Andersson, A. E., Fagerdahl, A.-M. y Knudsen, V. E. (2017). Images of suffering depicted in diariea IJMK, N- $\mathrm{s}$ of family caregivers in the acute stage of necrotising soft tissue infection: A content analysis. Intensive and Critical Care Nursing, 41, 57-62. Recuperado de: https:// doi.org/10.1016/j.iccn.2017.02.004

Enfermedades huérfanas. (s. f.). Recuperado de https://www.minsalud.gov.co/salud/publica/ PENT/Paginas/enfermedades-huerfanas.aspx

Fundación Universitaria de Ciencias de la Salud. Hospital de San José. Bogotá, Colombia., Casas, C., Hernández, S., Fundación Universitaria de Ciencias de la Salud. Hospital de San José, Helena Solano, M., Fundación Universitaria de Ciencias de la Salud. Hospital de San José, Castiblanco, R., Fundación Universitaria de Ciencias de la Salud. Hospital de San José, Carrillo, A., \& Fundación Universitaria de Ciencias de la Salud. Hospital de San José. (2018). Experiencias de mujeres frente al cuidado de hijos diagnosticados con hemofilia. IATREIA, 31(2), 145-154. Recuperado de: https:// doi.org/10.17533/udea.iatreia.v31n2a03

Harrington, M., Whalley, D., Twiss, J., Rushton, R., Martin, S., Huynh, L., y Yang, H. (2019). Insights into the natural history of metachromatic leukodystrophy from interviews with caregivers. Orphanet Journal of Rare Diseases, 14(1), 89. https://doi.org/10.1186/s13023-019-1060-2JAE v79n5.pdf. (s. f.). Recuperado de:http://circle. adventist.org/files/jae/JAE v79n5.pdf

Kavanaugh, M. S., Cho, C. C., \& Howard, M. (2019). "I Just Learned by Observation and Trial and Error": Exploration of Young Caregiver Training and Knowledge in Families Living with Rare Neurological Disorders. Child \& Youth Care Forum, 48(4), 479-492. Recuperado de: https:// doi.org/10.1007/s10566-019-09490-z

Kesselheim, A. S., McGraw, S., Thompson, L., O'Keefe, K., \& Gagne, J. J. (2015). Development and Use of New Therapeutics for Rare Diseases: Views from Patients, Caregivers, and Advocates. The Patient - Patient-Centered Outcomes Research, 8(1), 75-84. Recuperado de: https:// doi.org/10.1007/s40271-014-0096-6

Landfeldt, E., Edström, J., Buccella, F., Kirschner, J., y Lochmüller, H. (2018). Duchenne muscular dystrophy and caregiver burden: A systematic review. Developmental Medicine y Child Neurology, 60(10), 987-996. Recuperado de: https://doi.org/10.1111/dmcn.13934

Landfeldt, E., Lindgren, P., Bell, C. F., Guglieri, M., Straub, V., Lochmüller, H., y Bushby, K. (2016a). Health-related quality of life in patients with Duchenne muscular dystrophy: A multinational, cross-sectional study. Developmental Medicine y
Child Neurology, 58(5), 508-515. Recuperado de: https://doi.org/10.1111/dmcn.12938

Landfeldt, E., Lindgren, P., Bell, C. F., Guglieri, M., Straub, V., Lochmüller, H., \& Bushby, K. (2016b). Quantifying the burden of caregiving in Duchenne muscular dystrophy. Journal of Neurology, 263(5), 906-915. Recuperado de:https://doi.org/10.1007/ s00415-016-8080-9

Lauder, B., Sinclair, P. M., \& Maguire, J. (2018). Mothers' experience of caring for a child with early onset scoliosis: A qualitative descriptive study. Journal of Clinical Nursing, 27(7-8), e1549-e1560. Recuperado de: https://doi. org/10.1111/jocn.14301

Linertová, R., González-Guadarrama, J., SerranoAguilar, P., Posada-De-la-Paz, M., Péntek, M., Iskrov, G., \& Ballester, M. (2019). Schooling of Children with Rare Diseases and Disability in Europe. International Journal of Disability, Development and Education, 66(4), 362-373. Recuperado de: https://doi. org/10.1080/1034912X.2018.1562159

Magliano, L., Patalano, M., Sagliocchi, A., Scutifero, M., Zaccaro, A., D’Angelo, M. G., Civati, F., Brighina, E., Vita, G., Vita, G. L., Messina, S., Sframeli, M., Pane, M., Lombardo, M. E., Scalise, R., D’Amico, A., Colia, G., Catteruccia, M., Balottin, U., ... Politano, L. (2014). "I have got something positive out of this situation": Psychological benefits of caregiving in relatives of young people with muscular dystrophy. Journal of Neurology, 261(1), 188-195. Recuperado de:https://doi.org/10.1007/s00415-013-7176-8

Mori, Y., Downs, J., Wong, K., Anderson, B., Epstein, A., y Leonard, H. (2017). Impacts of caring for a child with the CDKL5 disorder on parental wellbeing and family quality of life. Orphanet Journal of Rare Diseases, 12(1), 16. Recuperado de: https://doi.org/10.1186/s13023-016-0563-3

NIC.pdf. (s. f.). Recuperado de http://www. consultadelsiglo21.com.mx/documentos/NIC.pdf

NOC.pdf. (s. f.). Recuperado de http://www. consultadelsiglo21.com.mx/documentos/NOC. pdf

OMS | Unidos para combatir las enfermedades raras. (s. f.). WHO. Recuperado de https://www.who.int/ bulletin/volumes/90/6/12-020612/es/

Palacios-Ceña, D., Famoso-Pérez, P., SalomMoreno, J., Carrasco-Garrido, P., Pérez-Corrales, J., Paras-Bravo, P., y Güeita-Rodriguez, J. (2018). "Living an Obstacle Course": A Qualitative Study Examining the Experiences of Caregivers of Children with Rett Syndrome. International Journal of Environmental Research and Public 
Health, 16(1), 41. Recuperado de: https://doi. org/10.3390/ijerph16010041

Pelentsov, L. J., Fielder, A. L., y Esterman, A. J. (2016). The Supportive Care Needs of Parents With a Child With a Rare Disease: A Qualitative Descriptive Study. Journal of Pediatric Nursing, 31(3), e207-e218. Recuperado de: https://doi. org/10.1016/j.pedn.2015.10.022

Pelentsov, L. J., Laws, T. A., \& Esterman, A. J. (2015). The supportive care needs of parents caring for a child with a rare disease: A scoping review. Disability and Health Journal, 8(4), 475491. Recuperado de: https://doi.org/10.1016/j. dhjo.2015.03.009

RESOLUCION-8430-DE-1993.pdf. (s. f.). Recuperado de https://www.minsalud.gov.co/sites/rid/Lists/ BibliotecaDigital/RIDE/DE/DIJ/RESOLUCION8430-DE-1993.PDF

Rice, D. B., Cañedo, M., Carboni, A., Carrier, M.E., Cumin, J., Malcarne, V. L., Hagedoorn, M., Thombs, B. D., y Scleroderma Caregiver Advisory Team. (2019). Challenges and support service preferences of informal caregivers of people with systemic sclerosis: A cross-sectional survey. Disability and Rehabilitation, 1-7. Recuperado de: https://doi.org/10.1080/09638288.2018.1557268

Schwartz, C. E., Powell, V. E., \& Eldar-Lissai, A. (2017). Measuring hemophilia caregiver burden: Validation of the Hemophilia Caregiver Impact measure. Quality of Life Research, 26(9), 25512562. Recuperado de:https://doi.org/10.1007/ s11136-017-1572-y

Soares, J. de L., Araújo, L. F. S. de, Bellato, R., Universidade Federal do Mato Grosso, Brazil, Universidade Federal do Mato Grosso, Brazil, y Universidade Federal do Mato Grosso, Brazil. (2016). Cuidar na situação de adoecimento raro: Vivência da família e sua busca por amparo dos serviços de saúde. Saúde e Sociedade, 25(4), 1017-1030. Recuperado de: https://doi. org/10.1590/s0104-12902016162301

Somanadhan, S., y Larkin, P. J. (2016). Parents' experiences of living with, and caring for children, adolescents and young adults with Mucopolysaccharidosis (MPS). Orphanet Journal of Rare Diseases, 11. Recuperado de: https://doi. org/10.1186/s13023-016-0521-0

Stewart, M., Shaffer, S., Murphy, B., Loftus, J., Alvir, J., Cicchetti, M., \& Lenderking, W. R. (2018). Characterizing the High Disease Burden of Transthyretin Amyloidosis for Patients and Caregivers. Neurology and Therapy, 7(2), 349364. Recuperado de:https://doi.org/10.1007/ s40120-018-0106-z
Tejada, E. M., Flores-Rojas, K., Moreno-Quintana, L., Muñoz-Villanueva, M. C., Pérez-Navero, J. L., y Gil-Campos, M. (2019). Necesidades sanitarias y socioeducativas de niños con enfermedades raras de tipo metabólico y sus familias: Estudio cualitativo en un hospital de tercer nivel. Anales de Pediatría, 90(1), 42-50. Recuperado de: https://doi.org/10.1016/j.anpedi.2018.03.003

Uttley, L., Carlton, J., Woods, H. B., \& Brazier, J. (2018). A review of quality of life themes in Duchenne muscular dystrophy for patients and carers. Health and Quality of Life Outcomes, 16(1), 237. Recuperado de: https://doi.org/10.1186/s12955018-1062-0

Yoo, J., Halley, M. C., Lown, E. A., Yank, V., Ort, K., Cowan, M. J., Dorsey, M. J., Smith, H., lyengar, S., Scalchunes, C., y Mangurian, C. (2019). Supporting caregivers during hematopoietic cell transplantation for children with primary immunodeficiency disorders. Journal of Allergy and Clinical Immunology, 143(6), 2271-2278. Recuperado jaci.2018.10.017

Recibido: 24/02/2020

Aceptado: 29/05/2020 